Supporting Information

\title{
Toehold-mediated strand displacement reaction for dual-signal electrochemical assay of apolipoprotein $\mathbf{E}$ genotyping
}

\author{
Hanwen $\mathrm{Lu}^{\mathrm{a}}$, Binrong Ding ${ }^{\mathrm{b}}$, Liujuan Tong ${ }^{\mathrm{a}}$, Fan $\mathrm{Wu}^{\mathrm{a}}$, Xinyao $\mathrm{Ya}^{\mathrm{a} *}$, Jianxiu Wang ${ }^{\mathrm{a} *}$ \\ ${ }^{a}$ Hunan Provincial Key Laboratory of Micro \&Nano Materials Interface Science, \\ College of Chemistry and Chemical Engineering, Central South University, Changsha, \\ Hunan 410083, P. R. China \\ ${ }^{b}$ Department of Geriatrics, The Third Xiangya Hospital of Central South University, \\ Changsha, Hunan 510060, P. R. China
}

*Corresponding author. E-mail: yixinyao@csu.edu.cn; jxiuwang@csu.edu.cn 

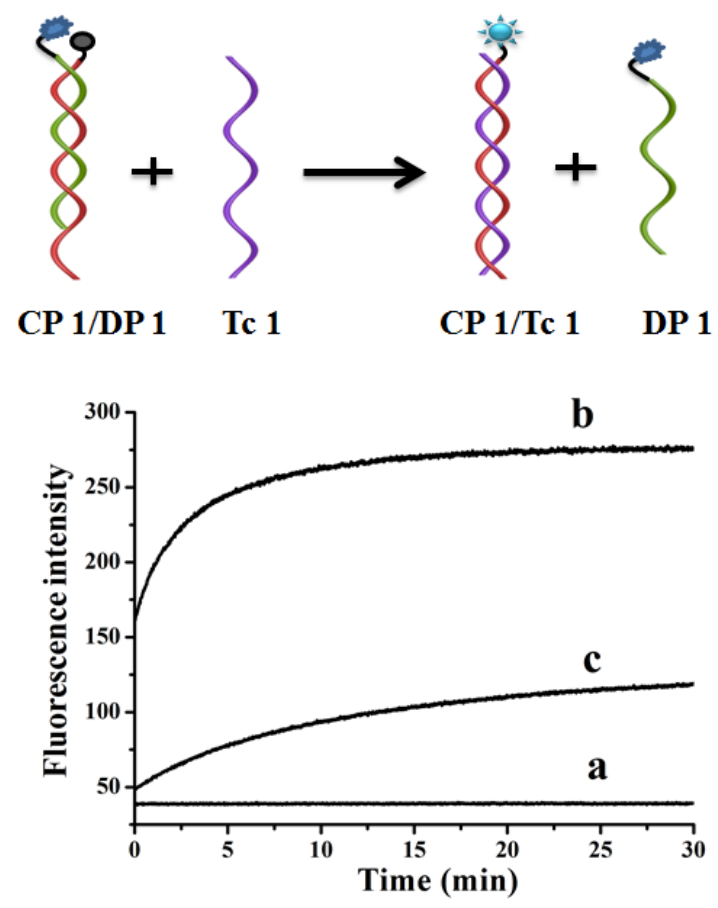

Figure S1. Real-time fluorescence measurement of the SDR. The concentrations of CP 1, DP 1, Tsm 1 and Tc 1 were all $500 \mathrm{nM}$.

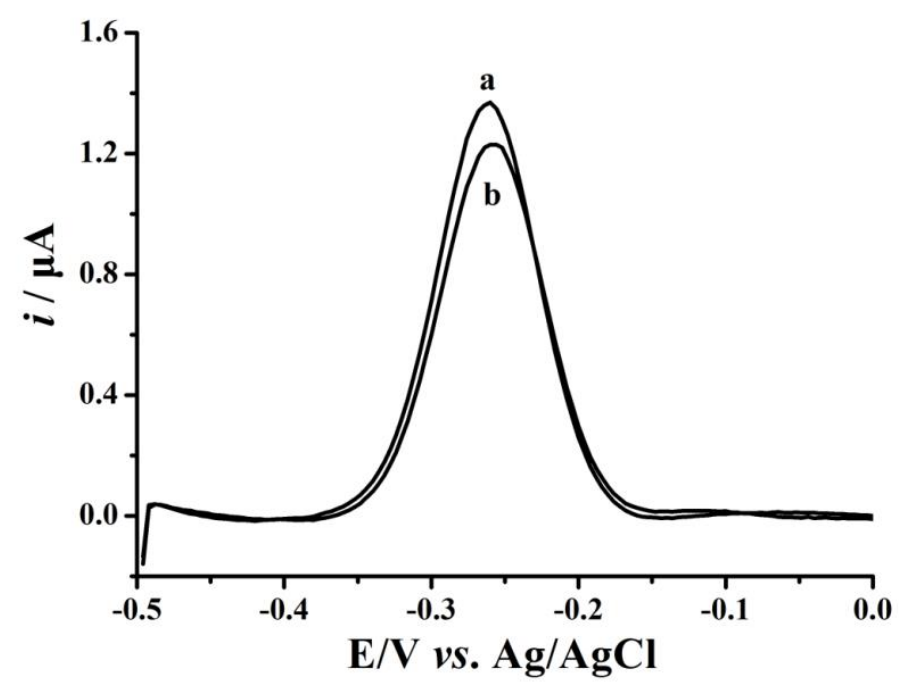

Figure S2. DPV response acquired at the electrode upon hybridization of the surface-confined CP 1 to MB-capped DP 1-modified AuNPs (a) and that via hybridization of the surface-confined CP 1 and 2 to MB-capped DP 1 - and MB-capped DP 2-modified AuNPs, respectively (b). The concentration of CP 1 was 1 $\mu \mathrm{M}$ in curve a, while those of $\mathrm{CP} 1$ and 2 in curve $\mathrm{b}$ were both $0.5 \mu \mathrm{M}$. 

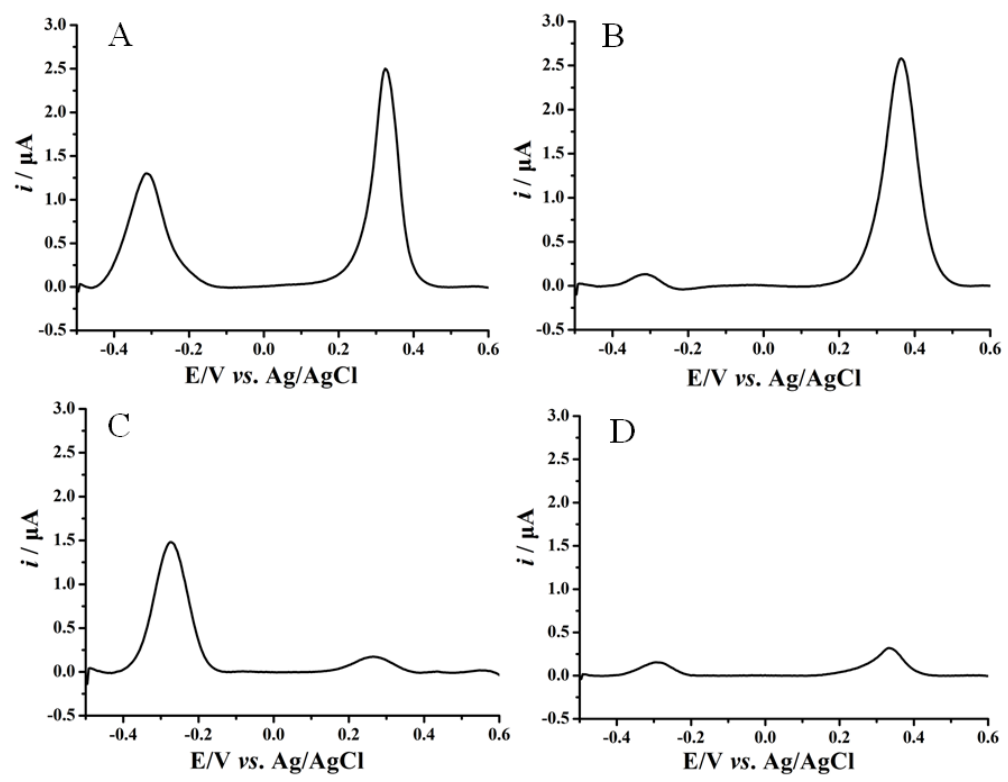

Figure S3. DPV responses acquired at the electrode upon hybridization of the surface-confined CP 1 and 2 to MB-capped DP 1- and Fc-capped DP 2-modified AuNPs, respectively (A), followed by displacement of the modified AuNPs by $5 \mathrm{pM}$ Tc 1 (B), 5 pM Tc 2 (C) or 5 pM Tc $1+5$ pM Tc 2 (D).

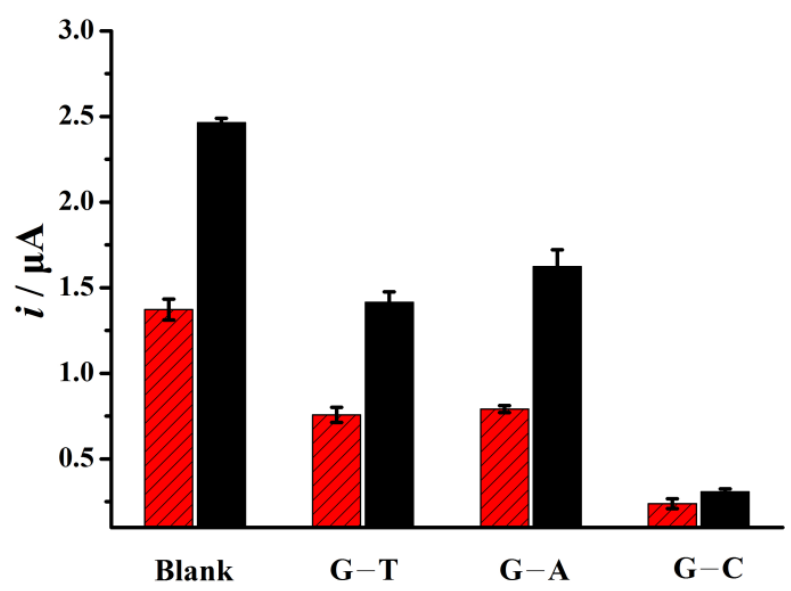

Figure S4. Specificity of apoE genotyping assay. The oxidation peak currents of MB and $\mathrm{Fc}$ tags (red and black columns, respectively) were measured in the cases of single-base mismatched sequences (G-T mismatch and G-A mismatch) and the complementary sequence of Tc 1 and Tc 2 (G-C match). The peak currents acquired upon hybridization of the surface-confined CP 1 and CP 2 with MB-capped DP 1- and Fc-capped DP 2-modified AuNPs, respectively, were shown as the blank. The concentrations of the assayed sequences were maintained at $5 \mathrm{pM}$. The absolute errors deduced from three replicate measurements were shown as the error bars. 